\title{
Les formateurs en institut de formation en masso-kinésithérapie : quelles conséquences pédagogiques de la réforme des études de 2015?
}

\section{Charlotte Pourcelot \\ Thérèse Perez-Roux \\ Eric Maleyrot}

Université Paul Valéry - Montpellier 3, France

\author{
The instructors in Institute of Training \\ in Physiotherapy: what are the pedagogical \\ consequences of the reform of the studies of 2015 ?
}

$\mathrm{R}$ ésumé

En France, les études de masso-kinésithérapie ont été réformées en 2015, elles mettent de l'avant une démarche réflexive et valorisent l'approche par problèmes et par compétences. Les formateurs impliqués sont logiquement confrontés à d'importants bouleversements remettant en question leurs perceptions de la formation, leurs stratégies d'intervention et leur professionnalisation. La présente étude s'intéresse aux équipes pédagogiques des Instituts de Formation en Masso-Kinésithérapie (IFMK) au travers des notions de rapport au savoir et d'expertise. Des focus group et une Analyse des Correspondances Multiples (ACM) ont été réalisés pour appréhender la manière dont les formateurs se saisissent de ces évolutions et se caractérisent.

Mots-clés

Formateurs, masso-kinésithérapie, réforme des études, rapport au savoir, expertise.

Abstract

In France, physiotherapist studies were reformed in 2015.

Thus, a combined reflexive approach, problem approach

and skill approach. Course instructors are confronted with numerous challenges and changes questioning the formation's perceptions, the way they intervene and the way they teach their students to become professionals. A study concerned the physiotherapy institute's teams as to better implement this transition. This is what we are trying to illustrate through notions of relationship to knowledge and expertise. Focus group and a Multiple Correspondence Analysis (ACM) were carried out to understand the way in which trainers grasp these developments and characterize themselves.

Keywords

Instructors, physiotherapy, educational reforms, relationship to knowledge, expertise.

\section{Contexte}

\section{Les évolutions vécues par les professionnels de la formation}

En France, au cours de ces dix dernières années, les formations des métiers adressés à autrui et à forte dimension relationnelle (enseignement, éducation, santé, travail social) ont été réformées. Les équipes directionnelles et pédagogiques ont vécu des évolutions institutionnelles et organisationnelles interrogeant leurs pratiques et troublant parfois leur sphère professionnelle (Perez-Roux, et Balleux, 2014). Ces transformations nous intéressent tout particulièrement en ce contexte de professionnalisation ${ }^{1}$ et d'universitarisation de la formation des Masseurs-Kinésithérapeutes (MK); c'est pourquoi cet article y est dédié, notamment sous l'angle des transformations pédagogiques.

\section{Les conséquences pédagogiques engendrées par les réformes des études}

Des travaux se sont intéressés aux tensions à l'œuvre entre professionnalisation et universitarisation dans les métiers de l'humain, parmi lesquels ceux de Ade et Piot (2018). Ils ont entre autres interrogé les tensions entre théorie et pratique et ont démontré que celles-ci engendraient des conséquences sur les méthodes pédagogiques des formateurs. En ce qui concerne les formations paramédicales, les formateurs en masso-kinésithérapie sont amenés à sortir d'une posture essentiellement transmissive des «bons gestes professionnels» vers une pédagogie stimulant la réflexivité des apprenants. Ce glissement est relayé par un appui sur les Sciences de l'Éducation et de la Formation. En effet, certains formateurs font le choix d'intégrer un cursus dans ce domaine pour renforcer leur professionnalité. Essentiellement fondée sur des situations complexes 
à traiter, cette pédagogie permet de rendre les étudiants plus actifs et de veiller à leur apprentissage en profondeur (Biggs, 1987).

L'étude présentée dans cet article s'intéresse à ce contexte de transition, et plus précisément aux formateurs en Institut de Formation en Masso-Kinésithérapie (IFMK) qui vivent une réingénierie de la formation depuis 2015 et qui ont dû modifier leur manière d'enseigner et les conditions d'apprentissage de leurs étudiants en adoptant une double logique de professionnalisation (Wittorski, 2007, 2008) et d'universitarisation (Bourdoncle, 2007) induite par le nouveau dispositif de formation. Pour saisir et appréhender les démarches et méthodes pédagogiques des formateurs en IFMK et les défis auxquels ils sont confrontés, nous nous intéresserons d'abord aux notions de rapport au savoir et d'expertise qui constituent les outils théoriques avec lesquels nous analyserons les tensions entre théorie et pratique.

\section{Cadre théorique}

\section{Le rapport au savoir, un concept essentiel pour lire le travail des formateurs en IFMK}

Depuis la promulgation de la réforme des études de masso-kinésithérapie, de nouvelles contraintes sont nées dans le cadre de la formation : le rapport au savoir des formateurs est réinterrogé au regard de leurs pratiques réelles d'enseignement et du nouveau plan de formation se présentant sous forme d'unités d'enseignement et de compétences attendues chez les étudiants. Les formateurs sont amenés à redéfinir en partie leur activité et à re(construire) le sens de leur travail (Perez-Roux et Balleux, 2014). Le rapport au savoir a ceci de particulier qu'il revêt un caractère générique et transdisciplinaire et qu'il s'inscrit dans différents champs de recherche que nous nous contenterons de citer : sociologique (Bourdieu, 1965), clinique (Beillerot, 1989), anthropologique (Chevallard, 1992), macro-sociologique (Hayder, 1997), microsociologique (Charlot, 1997), et didactique (Chartrain, 1998/Caillot, 1999). Ici, l'approche microsociologique développée par Charlot (1997) et qui représente «l'ensemble des relations qu'un sujet entretient avec un objet, un "contenu de pensée", une activité, une relation interpersonnelle, un lieu, une personne, une situation, une occasion, une obligation, etc., liés en quelque façon à l'apprendre et au savoir» (p. 94) fait écho à notre objectif de recherche. En effet, d'après Charlot (1997), un individu se construit grâce à autrui, aux objets culturels de savoir et à travers des projets personnels, des aspirations professionnelles et sociales. L'interaction entre le social et l'individuel dans la construction du rapport au savoir accentue le caractère dynamique et évolutif de ce concept auquel Charlot (1990) a pu attribuer trois dimensions : une dimension identitaire relative à l'origine du sens et de la valeur attribuée au savoir ; une dimension épistémique liée à la nature et la signification attribuée à l'apprentissage ; une dimension sociale transversale aux deux premières dimensions.

Sur cette base, nous postulons que ce qui est lié au-x savoir-s et plus largement à l'acte d'apprendre (connaître, comprendre, étudier, faire usage des savoirs, etc.) semble se modifier chez ces formateurs. En s'intéressant de plus près à la formation en masso-kinésithérapie, depuis la promulgation du décret et de l'arrêté du 2 septembre 2015 relatif au Diplôme d'État (DE) de Masseur-Kinésithérapeute (MK), les équipes directionnelles et pédagogiques ont eu à mettre en place une approche par compétences, en remplacement d'une approche centrée sur les contenus d'enseignements théoriques et pratiques (Maleyrot, Perez-Roux, Pourcelot, et Hébrard, 2019). 
La logique d'enseignement initialement centrée sur la transmission de savoirs académiques et pratiques et pensée avec une certaine progressivité n'est plus la même. La logique de formation est désormais axée sur l'acquisition des compétences dans des situations de travail et/ou de mises en situation plus complexes parce que plus proches de la réalité : être compétent, c'est pouvoir agir en contexte. Les étudiants sont ainsi appelés à devenir des professionnels compétents, capables « de mobiliser, de mettre en cuvre de façon efficace les différentes fonctions d'un système où interviennent des ressources aussi diverses que des opérations de raisonnement, des connaissances, des activations de la mémoire, des évaluations, des capacités relationnelles ou des schémas comportementaux» (Le Boterf, 1994, p. 43), et également des praticiens réflexifs (Schön, 1994) capables de rechercher des savoirs cachés dans leur agir professionnel.

Les formateurs sont amenés à accompagner ces étudiants en développant leur capacité réflexive les conduisant vers l'autonomie pour que, une fois diplômés, ils soient en mesure d'analyser toutes situations cliniques et de prendre des décisions adaptées. Pour les guider et faire appel à leur(s) expertise(s), le référentiel de formation comporte deux volets : le premier est composé des sciences fondamentales, des savoirs, savoir-faire disciplinaires et savoir-faire associés d'éducation et de rééducation en massokinésithérapie. Le deuxième vise à développer les aptitudes nécessaires au futur MK comme les gestes techniques de kinésithérapie et les aptitudes interpersonnelles.

Maintenant, intéressons-nous de plus près à la notion d'expertise dont l'importance semble être forte dans le monde des MK.

\section{La notion d'expertise en formation}

Bien qu'issue du monde de l'entreprise, tout comme les notions de «compétences " et de " professionnalisation », l'expertise des formateurs est au cœur de la formation des MK. Si c'est d'abord une notion qui s'oppose à celle de novice et que le duo expert-expérience semble ne faire qu'un, que nous apprend la documentation scientifique ? L'étymologie du mot « expert » rappelle que les compétences de ce dernier émanent de savoir-faire acquis grâce à l'expérience. D'ailleurs, l'expertus, qui provient du verbe experiri, est celui "qui a fait ses preuves, qui a de l'expérience, qui est habile» (Calafat, 2011). Selon Tardif et Lessard (1999), l'expérience de l'enseignant - et donc plus largement du formateur - renvoie aux «connaissances issues de l'expérience quotidienne du travail [qui] semblent [être] les fondements de la pratique du métier et de la compétence professionnelle ( (p. 370). Selon Bereiter et Scardamalia (1992), Darling-Hammond et Snyder (1992), Gustafsson et Undheim (1996), Munby, Russell et Martin (2001) ou encore Spencer (2001), l'enseignant expert posséderait des caractéristiques spécifiques et exclusives qui le distingueraient de l'enseignant débutant. "Ces attributs de l'expertise se dégageraient de l'expérience de l'enseignement, des pratiques effectives» (Lenoir, 2004).

Enfin, de l'expertise à l'acte d'enseigner, la Théorie de l'Action Conjointe en Didactique (TACD), qui comporte une dimension clinique, permet de comprendre et d'expliquer les fondations de l'action professorale que Sensevy (2007) appelle «déterminations du jeu». Pour l'enseignement de la massokinésithérapie, cette dimension peut être rapprochée du rapport au savoir et de la praxéologie de l'enseignant proposé par la Théorie Anthropologique du Didactique (TAD) (Chevallard, 1992). En

effet, tout enseignant entretient avec le savoir et avec le savoir enseigner, un ou des rapports personnels ou institutionnels, plus ou moins denses et plus ou moins conscients. 
Dans notre cas, cet aspect est d'autant plus prégnant puisque la masso-kinésithérapie n'est pas une discipline scolaire et qu'il existe dans le milieu des références variées.

À la suite de ces repères théoriques, nous nous interrogeons : quelle expertise les formateurs mettent-ils en avant ? Comment leur rapport au savoir se trouve-t-il transformé avec le changement de paradigme contenu-compétence/réflexivité ?

Pour exposer notre analyse autour de ces questions et dans une perspective exploratoire, nous proposons des éléments de réponse issus de focus group et d'un questionnaire.

\section{Méthodologie : double enquête au sein des IFMK}

\section{Une enquête par focus group dans un IFMK du Sud de la France}

L'enquête s'est déroulée dans un IFMK du Sud de la France (IFMK-S) dont le directeur a sollicité une équipe de chercheurs en Sciences de l'éducation pour être accompagné dans la mise en place de la réforme des études de MK de 2015.

Cet établissement recense une centaine de formateurs qui pour la plupart sont MK professionnels exerçant en secteur libéral ou public (salarié du Centre Hospitalier ou d'un établissement de rééducation) et qui enseignent souvent dans leur spécialité professionnelle puisque la majeure partie d'entre eux a une formation complémentaire au diplôme de MK (Diplôme Universitaire et/ou Master notamment). Dans une moindre mesure, interviennent également des médecins, des préparateurs physiques ou encore des psychologues.

Trois focus group ont donc été menés en janvier, mars et mai 2016, soit au cours de la première année de la réforme. Ceux-ci ont réuni douze formateurs volontaires au profil varié (hommes/femmes, emploi salarié/libéral, formateurs expérimentés/débutants, formateurs en centre/tuteurs de stage) qui ont pu s'exprimer sur le contenu et les effets de la réforme de 2015 sur la formation.

Une grille d'entretien composée de trois parties a servi à la conduite des focus group. Cette dernière abordait les préoccupations du moment, les problèmes et difficultés de mise en œuvre par les acteurs et enfin les éléments jugés intéressants dans la réforme. L'analyse de contenu a notamment permis de repérer les inquiétudes liées aux transformations pédagogiques et c'est sur ce thème que nous nous focaliserons dans cet article.

\section{Une enquête par questionnaire diffusée à l'échelle nationale}

À la suite de ces focus group, une enquête par questionnaire a également été lancée à l'échelle nationale pour savoir si les réponses apportées par les formateurs d'un seul IFMK étaient identiques dans les autres IFMK français. Par conséquent, les réponses apportées lors des focus group ont servi de base à la rédaction du questionnaire national. Composé de soixante-deux questions (fermées, à choix multiples ordonnés et ouvertes), il a été diffusé en ligne au printemps 2017 grâce à SphinxOnline. Il ciblait : le profil de MK et de formateur, le travail collectif au sein de l'IFMK, la fonction de formateur et de tuteur depuis la réforme, la perception de la réforme des études de MK de 2015 et la satisfaction ressentie. 
Pour l'analyse des résultats, nous avons opté pour une Analyse des Correspondances Multiples (ACM). Celle-ci convient parfaitement au traitement d'enquête par questionnaire lorsqu'il comporte des variables nominales parce qu'elle permet d'expliquer, à l'aide de données socio-administratives, des comportements ou des opinions. Les caractéristiques et profils de formateurs présentés ci-dessous émergent aussi d'un tri à plat et de tris croisés pour les questions fermées et à choix multiples. Elles permettent de découvrir les enquêtés sous l'angle de leur origine professionnelle, des formations qu'ils ont suivies ou dont ils auraient besoin, et de saisir leurs avis sur la réforme des études de 2015.

Les réponses aux questions ouvertes permettent quant à elles de mieux comprendre les conséquences de cette réforme sur leur fonction et les changements ressentis.

\section{Résultats}

Les résultats se présentent en deux parties : la première est dédiée aux focus group locaux et la seconde au questionnaire national.

\section{Les premières inquiétudes exprimées liées aux transformations pédagogiques}

Tout changement engendre des interrogations et des inquiétudes. Les focus group nous ont ainsi permis de révéler des appréhensions liées aux nouveaux modèles de formation à mettre en place. L'abandon des approches transmissives au profit de l'approche par problèmes, dans le but de développer la réflexivité des étudiants, est jugé pertinent, mais inquiétant par les formateurs. "Enseignant ou formateur, c'est un nouveau métier quion n'a pas appris, mais quion exerce en parallèle à celui de kiné. C'est une nouvelle fonction", déclare l'un d'eux.

Les formateurs s'interrogent aussi sur les contenus et les dispositifs à mettre en œuvre pour développer les nouvelles compétences attendues du MK et sur la posture du formateur et du tuteur de stage. «Les méthodes d'enseignement ont évolué, elles sont plus actives", rappelle un interviewé. Pour un autre, une perte de la technicité due à un manque de pratique est à craindre. "Le mémoire est important, il implique une capacité de réflexion accrue, mais il risque de "fabriquer des super cerveaux qui n'ont plus de mains" . En effet, "attention à une dérive intellectuelle au détriment des savoirs pratiques », dit-il alors que certains y voient une opportunité pour s'enrichir comme le démontrent ces deux témoignages : "au travers de l'accompagnement du mémoire, je me forme aussi ", "la recherche bibliographique permet une orientation vers des sujets que je n'ai pas le temps d'approfondir ».

Les focus group pointent de nombreux troubles et questions soulevées chez les enseignants au sujet du mémoire, étant donné que les formateurs et les tuteurs ne sont pas préparés à diriger des mémoires de recherche : comment diriger un mémoire de recherche sans y être soi-même acculturé ? À quelles exigences théoriques et méthodologiques devra-t-il répondre ? Comment se répartir les rôles entre formateur et tuteur ? Des interrogations concernent aussi le stage : quel est le rôle du maître de stage ? Quelle définition des objectifs de stage ? Quels documents de liaison entre le lieu de stage et l'école de masso-kinésithérapie ? Ces questionnements témoignent d'une remise en cause de leur expertise d'enseignant dans la nouvelle réforme et peut-être aussi de celle de MK, ou tout au moins la professionnalité attendue par la nouvelle prescription. 


\section{Les caractéristiques pédagogiques de la population formatrice en IFMK}

Avant d'aborder les caractéristiques pédagogiques des formateurs en masso-kinésithérapie et les défis auxquels ils sont confrontés, il convient de présenter brièvement notre population enquêtée à l'échelle nationale. L'échantillon $(\mathrm{n}=141)$ est plutôt masculin $(60 \%)$ et multi-âge. Près d'un tiers des répondants est âgé entre 41 et 50 ans (29\%) et les moins de 30 ans sont très peu nombreux (6\%). La quasi-totalité possède un diplôme de MK (94\%) et près de la moitié est titulaire d'un Master (46 \%). Un peu plus de deux sur cinq sont cadres de santé (42\%) et un quart exerce leur activité de MK en libéral (25\%).

\section{Les principales difficultés rencontrées}

Les enquêtés rencontrent trois grands types de difficultés. Près de la moitié déclare devoir faire face à des difficultés didactiques ${ }^{2}(44 \%)$ ainsi qu'à des complications liées à leur activité professionnelle comme un emploi du temps trop chargé ou une difficulté à se libérer (42\%). Un tiers éprouve aussi des difficultés professionnelles telles que faire comprendre les enjeux, les pratiques et les problématiques du métier (35\%).

Les enquêtés déclarent que la démarche réflexive avec les étudiants (47\%), le suivi du mémoire professionnel (40\%), et le travail de tuteur durant les stages (35\%) sont problématiques. La réflexivité est donc un levier majeur de la réforme, mais elle représente également une difficulté pour les équipes pédagogiques qui ne savent pas nécessairement la mettre en œuvre. Ainsi $53 \%$ des MK généralistes, $53 \%$ des MK spécialisés, $50 \%$ des cadres et $43 \%$ des formateurs à temps plein pensent que la démarche réflexive est délicate à instaurer. Les $\mathrm{MK}$ généralistes, les formateurs à temps plein et les cadres, sont entre 41 et $47 \%$ à déclarer que le suivi du mémoire professionnel est problématique alors que les MK spécialisés sont $28 \%$.

Un formateur (MK-Ostéopathe âgé entre 41 et 50 ans, formateur à l'IFMK depuis 3 à 5 ans) confie ne pas toujours maîtriser le contenu de ses cours théoriques et pratiques et manquer de compétences pédagogiques. "Je note ainsi l'importance notamment des supports pédagogiques bien construits pour garder un fil conducteur et en ce sens je manque de formation ", dit-il. Un second (MK cadre de santé âgé de plus de 60 ans, formateur à l'IFMK depuis plus de 40 ans) déclare qu' "enseigner la clinique et l'observation des pathologies » est une tâche difficile. Face aux étudiants, les formateurs peuvent se sentir démunis parce qu'ils ne possèdent pas suffisamment de connaissances relatives aux prérequis des étudiants. Ils peuvent alors avoir le sentiment de perdre de leur légitimité, comme ce formateur (MK cadre de santé âgé de plus de 60 ans, formateur à l'IFMK depuis plus de 10 ans) qui affirme que «lors de cours pratiques, en étant "sorti" de la profession depuis plus de 10 ans, les limites de la légitimité [se font ressentir] ».

La difficulté à concilier activité professionnelle et activité d'enseignement et de formation est également formulée par les formateurs à temps partiel. L'un rencontre des difficultés à allier ses métiers de MK et de formateur, visiblement par manque de disponibilité puisqu'il déclare : "Je suis salarié dans un centre de rééducation et chaque fois que je m'absente pour aller donner des cours, je suis contraint de répartir mes patients sur les collègues, augmentant ainsi leur charge de travail. Mais aussi je dois poser des jours de congés ou de récupération, ce qui me coûte à la longue. Cela me rend moins disponible que je le soubaiterais... (MK libéral âgé entre 31 et 40 ans, formateur à l'IFMK depuis plus de 6 ans).

Certains formateurs expriment aussi une complémentarité de leurs activités de MK et de formateur : 
"Mon activité de MK nourrit mon activité d'enseignante et en retour le développement de compétences engendré par cette activité nourrit mon activité de MK. Il me paraitrait inconcevable de ne plus enseigner» (MK-Ostéopathe âgée entre 31 et 40 ans, formatrice à l'IFMK depuis 6 à 10 ans). Cette "évolution permanente et remise en question » (MK libéral âgé en 31 et 40 ans, formateur à l'IFMK depuis 6 à 10 ans) semble alors bénéfique pour les étudiants. Or, le double métier ne facilite pas la communication et la coopération entre formateurs. Quelques formateurs relatent en effet un manque de communication au sein de leur équipe pédagogique. Ils relèvent des «difficultés de communication entre des intervenants sur un thème commun, menant les étudiants à avoir différents sons de cloche " (MK salariée du public âgée entre 31 et 40 ans, formatrice à l'IFMK depuis 3 à 5 ans) et "un manque de concertation entre enseignants » (MK cadre de santé âgé de plus de 60 ans, formateur à l'IFMK depuis 31 à 40 ans) pouvant créer des doublons.

L'expression de ces difficultés ouvre une fenêtre sur les formations suivies par les enquêtés pour exercer leur fonction.

\section{Le suivi de formations liées à l'activité de formateur}

Toutes formations confondues (enseignement, recherche, pédagogie, évaluation, analyse de la pratique professionnelle, tutorat, Technologies de l'Information de la Communication - TIC), les moins formés sont les MK généralistes (67\%). La grande majorité des enquêtés a reçu au moins une formation liée à l'enseignement (86\%) et notamment : pédagogique (52\%), aux méthodes d'enseignement (47\%), au tutorat et à l'accompagnement des étudiants en stage (40\%), à la méthodologie de recherche (38\%), à l'évaluation (38 \%) et à l'analyse des pratiques professionnelles (33\%).

Bien que la majorité des enquêtés soit formés au métier de formateur, ceux-ci expriment différents besoins liés à la réforme des études.

\section{Les besoins en formation exprimés}

Les répondants éprouvent des besoins en formation et notamment pour la recherche (31\%), les TIC (31\%), et les méthodes d'enseignement (28\%).

Quatre MK spécialisés sur dix souhaitent être formés aux méthodes d'enseignement (44\%) contre $21 \%$ des formateurs dont la formation représente l'activité principale. Plus du tiers des formateurs à temps plein souhaitent être formés à la recherche $(37 \%)$. Ces derniers sont quatre sur dix demandeurs de formation pour les TIC (40\%), alors que les MK généralistes et les MK spécialisés sont moins de deux sur dix à solliciter cette formation (respectivement $7 \%$ et $19 \%$ ). Une formatrice (MK salariée du public âgée entre 51 et 60 ans, formatrice à l'IFMK depuis 3 à 5 ans) exprime très clairement un besoin d'être initiée aux méthodes pédagogiques et d'accompagnement puisqu'il lui «manque des connaissances en méthodologie de recherche dans l'accompagnement des mémoires ». Le besoin d'être informé est également soulevé par l'un des formateurs sondés (MK-Ostéopathe diplômé d'un Master en Sciences de l'éducation, formateur à l'IFMK depuis plus de 11 à 20 ans), notamment sur la réforme des études de kinésithérapie, car la « réforme des études et [1'] interprétation des textes » sont problématiques.

87 - Formation et profession 28(3), 2020 


\section{Les défis de la réforme}

Les difficultés pédagogiques rencontrées, le besoin en formation de la tranche d'âge (51-60 ans) et la formation aux TIC font partie des défis à relever. La réflexivité est le point de la réforme le plus délicat à mettre en œuvre. Ils s'inquiètent donc de cette orientation. Les formateurs estiment que la démarche réflexive (94\%), l'approche par problèmes (43\%), et les nouveaux outils pédagogiques proposés par l'IFMK (32\%) sont des points forts de la réforme des études. Ces derniers constituent autant de défis à relever pour ceux-ci. Concrètement, les formateurs qui sont aussi tuteurs remarquent des changements. "Les étudiants sont plus orientés vers la recherche. Les tuteurs sont davantage amenés à les faire se justifier sur leurs actions ", témoigne cette formatrice (MK cadre de santé âgée entre 41 et 50 ans, formatrice à l'IFMK depuis 1 à 2 ans).

Sur le terrain, les étudiants font "preuve d'un meilleur niveau théorique et se posent plus de questions" (MK-Ostéopathe âgé entre 31 et 40 ans, formateur à l'IFMK depuis 3 à 5 ans), ils sont «de plus en plus impliqués» (MK libéral âgé entre 31 et 40 ans, formateur à l'IFMK depuis 3 à 5 ans), ils ont "une manière de réfléchir qui est différente» (MK salariée du public âgée entre 31 et 40 ans, formatrice à l'IFMK depuis 3 à 5 ans), et ils sont "plus intéressés, plus sérieux quant à la façon d'apprébender le lieu de stage " (MK salariée du public âgée entre 41 et 50 ans, formatrice à l'IFMK depuis 11 à 20 ans), ce qui peut être interprété comme une inquiétude quant à leur compétence et un défi à relever pour se « mettre à niveau » dans leur enseignement et accompagnement sur le terrain. À l'inverse, certains formateurs remarquent qu'ils sont "malheureusement plus dans l'analyse et moins dans le geste juste, en oubliant qu'ils sont devant de vrais patients » (MK libéral âgé de plus 60 ans, formateur à l'IFMK depuis plus de 11 à 20 ans), qu'ils sont "plus passifs et attendent souvent quion donne toutes les réponses à leurs questions [et] ils sont curieux, mais pas encore autonomes pour chercher leurs réponses seuls " (MK salariée du public âgée de moins de 30 ans, formatrice à l'IFMK depuis 1 à 2 ans). L'un d'eux affirme même que "la mise en cuvre de la réflexivité exigée par la réforme ne rencontre pas un soutien sur les terrains qui nén comprennent pas les enjeux pour la professionnalisation » (MK salarié du public diplômé d'un Master en Sciences de l'éducation, âgé entre 51 et 60 ans, formateur à l'IFMK depuis 11 à 20 ans).

Concernant l'activité principale, un formateur à temps plein sur deux (48\%) estime que l'approche par problèmes est un point positif de la réforme, de même que $42 \%$ des cadres, $40 \%$ des MK généralistes et $34 \%$ des MK spécialisés. Puis, quatre MK spécialisés sur dix (41\%), suivis de trois formateurs à temps plein sur dix (30\%) puis d'un quart des MK généralistes (27\%) et des cadres (25\%) déclarent que les nouveaux outils pédagogiques proposés par l'IFMK sont un point fort de la réforme.

Concernant la réforme, la majorité des formateurs la perçoivent comme une opportunité de développement (68\%). Selon les enquêtés, les aspects prioritaires de leur activité d'enseignement sont : accompagner l'étudiant dans son développement professionnel (46\%), susciter le plaisir de se former et de se professionnaliser (45\%), réfléchir sur ses pratiques d'enseignement et analyser leurs effets (32 $\%$, permettre d'accéder à des valeurs et à une culture professionnelle (31\%), et maîtriser et transmettre des savoirs théoriques jugés utiles pour un professionnel (26\%).

Mais la réforme, encore récente au moment de l'enquête, invite les formateurs à la prudence, voire à une certaine réticence. Une formatrice (MK cadre de santé âgée entre 31 et 40 ans, formatrice à l'IFMK depuis 1 à 2 ans) s'inquiète d'un possible «clivage entre anciens diplômés et nouveaux diplômés » et un second redoute une "perte des savoir-faire » due à «la distanciation des stages au cours du cursus, 
[car] un stage par semestre c'est peu... ». Une autre (MK cadre de santé âgée entre 31 et 40 ans, formatrice à l'IFMK depuis 1 à 2 ans) craint de "trop se focaliser sur la recherche et perdre le cour de métier ", voire de former de "futurs professionnels non efficaces voire dangereux en pratique, car n'ayant pas mesuré les conséquences des gestes thérapeutiques". Deux autres regrettent que "la redéfinition claire de la profession de masseur-kinésithérapeute n'ait pas été menée de façon assez profonde avant cette réforme " (MK cadre de santé âgé entre 51 et 60 ans, formateur à l'IFMK depuis plus de 6 ans), et pour la profession, ils déplorent la non-reconnaissance du "grade Master et [de] l'équivalence statutaire et salariale » et "le risque de déprofessionnalisation pour certains enseignants formateurs » (MK cadre de santé âgé entre 51 et 60 ans, formateur à l'IFMK depuis plus de 21 ans).

$\mathrm{Au}$ terme de ces résultats et témoignages, nous avons souhaité poursuivre notre analyse en effectuant une ACM. Celle-ci a permis de classer la population formatrice en IFMK.

\section{Trois classes de formateurs en IFMK}

La figure 1 représente les trois classes de formateurs en IFMK. Chaque individu y est représenté par le chiffre et la couleur de la classe à laquelle il appartient. Les modalités actives sont symbolisées par un carré rouge plein et les modalités illustratives par un carré vide. La variable « QE Expert », par exemple, correspond à une qualité essentielle d'un formateur en IFMK, ici l'expertise. Enfin, d'un point de vue méthodologique et dans le respect de cette méthode factorielle, des extraits d'interviews ne peuvent être dévoilés.

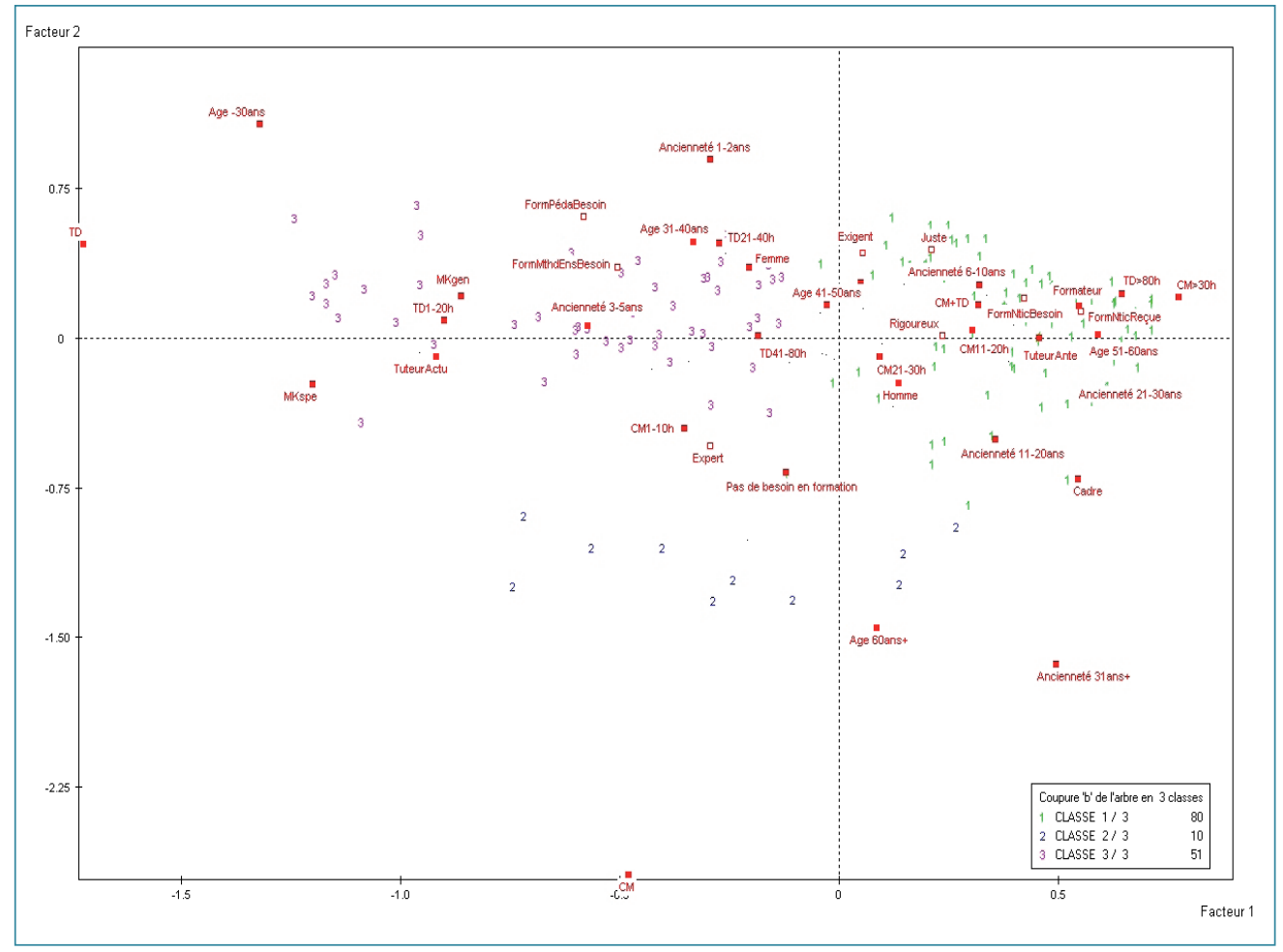

Figure 1

Représentation graphique de l'analyse factorielle en trois classes de la population formatrice en IFMK 


\section{Classe 1 : Les formateurs experts à plein temps}

Cette classe représente plus de la moitié de notre échantillon (effectif $80-57 \%$ ). Ces formateurs à temps plein dispensent un grand nombre d'heures de CM (plus de 30 heures) et de TD (plus de 80 heures). Ils ont très souvent un statut de cadre et un diplôme de cadre de santé. Ils ont suivi de nombreuses formations en rapport avec l'enseignement. Ils accompagnent les étudiants et encadrent les mémoires. Ils sont associés aux travaux entre formateurs (cours et pédagogie). Ils ne sont pas tuteurs actuellement, mais considèrent que le tutorat est un point délicat de la réforme.

\section{Classe 2 : Les intervenants seniors ponctuels}

Cette classe représente moins d'un individu sur dix, mais révèle des caractéristiques intéressantes pour notre analyse (effectif $10-7 \%$ ) puisqu'ils ne peuvent naturellement rejoindre aucune des deux autres classes. Ces formateurs ont plus de 60 ans et viennent d'autres professions (médecins notamment). Ils interviennent uniquement en CM avec un faible nombre d'heures (entre 1 et $10 \mathrm{~h}$ par an). Ces seniors pensent que la qualité essentielle d'un formateur en IFMK est l'expertise sur une ou plusieurs spécialités médicales.

\section{Classe 3 : Les jeunes MK-formateurs et tuteurs sans formation à l'enseignement}

Cette classe représente un tiers de notre échantillon (effectif 41 - $36 \%$ ). Ces formateurs à temps partiel sont aussi souvent tuteurs. Ils sont pour la plupart MK libéraux spécialisés ou généralistes et parfois ostéopathes. Ils sont plutôt jeunes (les moins de 30 ans sont surreprésentés dans la classe) et assurent un faible nombre d'heures de TD (entre 1-20h), et peu de CM. Ils ne sont pas associés aux travaux collectifs de leur établissement dont ils ne connaissent pas la nature.

$\mathrm{Au}$ terme de cette analyse, formulons quelques pistes de réflexion, hypothèses et perspectives de recherche sur la formation en pédagogie des formateurs en IFMK.

\section{Pistes de réflexion et propos conclusifs sur la formation en pédagogie des formateurs en IFMK}

L'analyse que nous avons menée questionne l'activité des formateurs, les stratégies de recrutement des équipes directionnelles et l'information et la formation des MK-tuteurs. Elle interroge aussi les parcours professionnels. En effet,le nouveau référentiel de formation des études de masso-kinésithérapie possède en filigrane une réflexion sur un enjeu formatif fondamental - l'acte d'apprendre - parce qu'il sous-tend le passage d'une démarche monstrative à la mise en œuvre de l'approche par compétences et de l'approche par problèmes; celles-ci supposent de mobiliser des méthodes actives (études de cas, projets d'étudiants, etc.) dans le but de favoriser le transfert des apprentissages vers la pratique professionnelle des apprenants. De nouvelles formes de formation et de réflexion par l'écrit comme le rapport d'étonnement (Thievenaz, 2014) sont mises en place au sein des IFMK et de nouveaux dispositifs à dimension innovante, réflexive et relationnelle sont amenés à se développer, et notamment la simulation de situations professionnelles réelles. 
Pour les trois classes de formateurs décrites dans cet article, la transition induite par la réforme des études se révèle différemment problématique, notamment d'un point de vue pédagogique, hormis pour les « formateurs seniors » qui semblent plutôt détachés. À notre connaissance, le rapport au savoir du formateur a, jusqu'à présent, fait état de peu de recherches. Il serait donc intéressant de mieux savoir de quoi il est formé et de mieux connaître les mécanismes à l'œuvre. Une étude comparative avec un autre groupe de formateurs pourrait par exemple être menée.

Projetée dans un univers complexe et évolutif, cette étude révèle aussi l'émergence d'une polyvalence qui ne se limite plus à l'expertise et qui peut être médicale, paramédicale ou encore pédagogique. Cette évolution devrait se traduire notamment par un changement de posture qui induit, entre autres, un transfert de compétences, une (ré)ingénierie portant sur les contenus des enseignements, une appropriation des fonctions d'animateur ou d'accompagnateur. Elle nécessiterait par ailleurs une compétence au travail collectif, indispensable pour mettre en œuvre, de façon concertée et efficiente, les attendus de la réforme. Dans une logique de formation, les équipes pédagogiques sont sollicitées en vue de développer les compétences de leurs apprenants sur la base de savoirs issus à la fois de la pratique en masso-kinésithérapie, des champs médicaux et de la recherche en santé et en SHS. Or, dans notre enquête, l'expertise kinésithérapique est citée comme qualité essentielle d'un «bon » formateur à l'IFMK. La réforme des études fait donc le lit d'une nécessaire transformation pédagogique, mais aucun accompagnement ne semble leur être proposé. Pourtant, certains expriment des besoins de formation aux méthodes d'enseignement et de recherche. Une fenêtre s'ouvre sur l'apport des Sciences de l'éducation à ces "professionnels-experts » dont la spécialisation dans un domaine particulier semble définir leur véritable identité de formateur. Dans le même temps, nous pouvons observer que l'universitarisation des formations paramédicales accorde une place renforcée à la pédagogie innovante, à la réflexivité et à la recherche. Les directions des instituts de formation sont alors confrontées à deux tendances opposées : 1) favoriser le parcours et le domaine universitaire du formateur qui exercera à temps complet et qui pourra s'appuyer sur ses compétences analysées au regard de travaux de recherche, 2) privilégier le « double métier » à travers l'embauche à temps partiel de professionnels de terrain (Bourdoncle, 2007).

D'autre part, la formation des enquêtés est réelle puisque les trois quarts de notre échantillon ont été formés au moins une fois en pédagogie. On peut noter que les formateurs les plus formés à l'enseignement sont aussi les plus diplômés (DU, Licence, Master et Doctorat) et que les formateurs qui possèdent pour plus haut diplôme celui de MK sont les moins formés. Les directions d'IFMK semblent avoir fait le choix de constituer des équipes pluri-catégorielles composées d'expertises diverses. Les classes de formateurs mises en exergue dans notre analyse montrent que les préoccupations pédagogiques sont variées et que les défis auxquels ceux-ci sont confrontés s'avèrent également bien différents. Notre étude montre également que de plus en plus de formateurs - exerçant en IFMK et proches de ceux-ci - ont pour projet de retourner en formation, notamment en Master de l'éducation et de la formation, portés par les universités ou les Instituts Nationaux Supérieurs du Professorat et de l'Éducation (INSPE), dans le but de s'acculturer à un autre mode de pensée et de réflexion. Devant l'élévation des savoirs théoriques et des compétences réflexives ainsi que les changements entre la logique de l'enseignement et la logique de la formation que certains formateurs souhaitent assumer, à l'issue de cette analyse, nous suggérons de poursuivre ces réflexions au travers d'une enquête qualitative en suivant des enseignants de chacune des trois classes identifiées. 
Finalement, plus que des réponses, il s'agit là d'une piste de recherche que nous formulons, car les enquêtes initiées jusqu'à présent sont selon nous à percevoir comme une base à consolider et à enrichir pour prolonger cette enquête exploratoire et, à l'appui de travaux récents (Perez-Roux, 2019 ; Maleyrot, Perez-Roux, Pourcelot, et Hébrard, 2019), rendre compte d'un processus de professionnalisation (Bourdoncle, 1991) pour les formateurs en IFMK.

\section{Notes}

1 Rappelons que le processus de professionnalisation est soutenu par la formation et le travail qui peut se définir comme un « processus de construction des connaissances, savoirs et identités reconnus comme faisant partie de la profession choisie » (Wittorski, 2007, p. 3).

2 Les 3 premiers items proposés sur les 11 relatifs aux difficultés rencontrées relevaient du cœur de la fonction de formateur : d'ordre didactique (choix et adéquation des contenus aux étudiant(e)s, de la progressivité des apprentissages); d'ordre pédagogique (gestion de certains groupes, public étudiant hétérogène); d'ordre professionnel (faire comprendre les enjeux, les pratiques et les problématiques du métier).

\section{Références}

Ade, D. et Piot, T. (2018). La formation entre universitarisation et professionnalisation. Tensions et perspectives dans des métiers de l'interaction humaine. Rouen, France : Presses Universitaires de Rouen et du Havre.

Beillerot, J. (2000). Le rapport au savoir. Dans J. Beillerot, N. Blanchard-Laville et N. Moscon (dir.), Formes et formations du rapport au savoir (p. 39-57). Paris, France : L'Harmattan.

Bereiter, C., et Scardamalia M. (1992). Cognition and curriculum. Dans P. W. Jackson (dir.), Handbook of research on curriculum (p. 517-542), New York, USA : Macmillan.

Biggs, J.-B. (1987). Student Approaches to Learning and Studying. Hawthorn, Australy : Australian Council for Educational Research.

Bourdoncle, R. (1991). La professionnalisation des enseignants : analyses sociologiques anglaises et américaines. Revue française de pédagogie, 94, 73-92.

Bourdoncle, R. (2007). Autour du mot : Universitarisation. Recherche et Formation, 54, 135-149.

Calafat, G. (2011). Expertise et compétences. Procédures, contextes et situations de légitimation. Hypothèses, 14, 95-107.

Charlot, B. (1990). Enseigner, former : Logique des discours constitués et logique des pratiques. Recherche E̊ Formation, $8(1), 5-17$.

Charlot, B. (1997). Du rapport au savoir. Paris, France : Anthropos.

Chevallard, Y. (1992). Concepts fondamentaux de la didactique : perspectives apportées par une approche anthropologique. Recherches en Didactique des Mathématiques, 12(1), 73-112.

Darling-Hammond L., et Snyder, J. (1992). Curriculum studies and the tradition of inquiry: The scientific tradition. Dans P.W. Jackson (dir.), Handbook of research on curriculum (p. 41-78), New York, USA : Macmillan.

Dubar, C. (2000). La crise des identités. L'interprétation d'une mutation. Paris, France : Presses Universitaires de France.

Gustafsson, J.-E., et Undheim, J.-O. (1996). Individual differences in cognitive functions. Dans D.C. Berliner et R.C. Calfee (dir), Handbook of educational psychology (p. 186-242), New York, USA : Macmillan.

Le Boterf, G. (1994). De la compétence : essai sur un attracteur étrange. Paris, France : Les Editions d'Organisation.

Lenoir, Y. (2004). L'enseignant expert : regard critique sur une notion non dépourvue d'intérêt pour la recherche sur les pratiques enseignantes. Recherche et Formation, (47). 
Maleyrot, E., Perez-Roux, T., Pourcelot, C., et Hébrard, P. (2019). Comprendre le travail de mise en œuvre de la réforme des études en Masso-Kinésithérapie (2015) : le cas d'un institut de formation. Activités, 16(1).

Perez-Roux, T. et Balleux, A. (2014). Introduction : Transitions professionnelles désiréescontraintes : quelles dynamiques identitaires des acteurs à l'épreuve des contexts? L'orientation Scolaire et Professionnelle, 43(4), 391-397.

Perez-Roux, T. (dir.). (2019). La réforme des études en santé entre universitarisation et professionnalisation : le cas des Instituts de Formation en Masso-Kinésithérapie. L'Harmattan : collection Pratiques en formation.

Schön, D.-A. (1994). Le praticien réflexif : à la recherche du savoir caché dans l'agir professionnel. Montréal, Canada : Les Editions Logiques.

Sensevy, G. (2007). Des catégories pour décrire et comprendre l'action didactique. Dans G. Sensevy et A. Mercier (dir.), Agir ensemble : Éléments de théorisation de l'action conjointe du professeur et des élèves (p. 13-49). Rennes : Presses Universitaires de Rennes.

Spencer, D. A. (2001). Teachers'work in historical and social context. Dans V. Richardson (dir.), Handbook of research on technopôle (p. 803-825) Washington, USA : American Educational Research Association.

Tardif, M., et Lessard, C. (1999). Le travail enseignant au quotidien. Contribution à l'étude du travail dans les métiers et les professions d'interactions humaines. Québec, Canada : Presses de l'Université Laval.

Thievenaz, J. (2014). Le rôle de l'étonnement dans l'apprentissage : contribution à l'approche par l'activité en formation des adultes. TransFormations, 11, 83-100.

Wittorski, R. (2008). Professionnaliser la formation : enjeux, modalités, difficultés. Formation Emploi, 101, 105-117.

Wittorski, R. (2007). Professionnalisation et développement professionnel. Paris, France : L'Harmattan.

\section{Pour citer cet article}

Pourcelot, C., Perez-Roux, T. et Maleyrot, E. (2020). Les formateurs en institut de formation en masso-kinésithérapie : quelles conséquences pédagogiques de la réforme des études de 2015. Formation et profession, 28(3), 81-93. http://dx.doi.org/10.18162/fp.2020.524 